\title{
Communication-free Voltage Regulation in Distribution Networks with Deep PV Penetration
}

\author{
Rayan El Helou \\ Texas A\&M University \\ rayanelhelou@tamu.edu
}

\author{
Dileep Kalathil \\ Texas A\&M University \\ dileep.kalathil@tamu.edu
}

\author{
Le Xie \\ Texas A\&M University \\ le.xie@tamu.edu
}

\begin{abstract}
In this paper, we present a scheme of fully localized voltage regulation in distribution grids through reactive power compensation using photovoltaic $(P V)$ inverters. We employ quasi-steady state representation of the effect of incremental changes in reactive power on voltage, in the form of discrete-time dynamics. We prove using this representation that nodal voltages may be regulated with guaranteed stability in the sense of Lyapunov without any node-to-node communication. We present properties of this communication-free control, such as guarantees on when it works and when it fails. Numerical studies based on realistic distribution network parameters are conducted to illustrate the performance and robustness of this proposed method with respect to changes of operating conditions and system parameters.
\end{abstract}

\section{Nomenclature}

$p_{i}^{\mathrm{PV}}(t) \quad$ Active power injected/provided by $\mathrm{PV}$ at node $i$, at time $t$

$q_{i}^{\mathrm{PV}}(t) \quad$ Reactive power injected by $\mathrm{PV}$ inverter (input) at node $i$, at time $t$

$v_{i}(t) \quad$ Squared voltage (output) at node $i$, at time $t$

$\mathbf{e}_{\boldsymbol{\Omega}}(t) \quad$ Voltage tracking error vector at time $t$, corresponding only to nodes in set $\boldsymbol{\Omega}$

$g_{i} \quad$ Feedback gain (control policy) at node $i$

$\theta_{i} \quad$ Risk factor adopted at node $i$

C.F. $\Omega$ Communication-free system where $g_{i}=0$ (inactive) for all nodes $i \notin \Omega$.

$X_{\Omega} \quad$ System parameter sub-matrix, with rows and columns corresponding only to nodes in set $\Omega$

$G_{\Omega} \quad$ Diagonal control gain matrix corresponding only to nodes in set $\boldsymbol{\Omega}$.

\section{Introduction}

Distribution power grids are experiencing increasing levels of solar photovoltaic (PV) penetration. One of the key operational challenges is how to regulate system-wide voltage performance over a wide range of net load variations. While PV introduces much intermittency in real power production, the inverters amounted alongside with the PV panels offer a tremendous opportunity to conduct voltage regulation in distribution systems. Compared with conventional voltage regulation through capacitor banks at the substations, PV inverters offer a much faster response and a possible localized solution [1-3]. Moreover, the localized voltage regulation through PV inverters are less vulnerable to cyberattacks under limited communication, and in this paper, we propose a controls framework that eleminates the need for communication entirely.

Recent work [4-7] focus on voltage control of radial networks under limited communication, and present objectives for optimal control. These results heavily rely on a communication layer between neighboring nodes in the grid. The appealing features of these results are that they are robust to communication delay and parameter estimation error. In [8], the model of the inverter capacity is updated from quadratic constraints to linear constraints, and an uncertainty model is provided for the local active power injections by the PV. It is suggested in IEEE Standard 1547-2018 to use droop control as a default scheme for VAR control. This method is tested in [9], and an improved scaled VAR control scheme is introduced with results that show better convergence of local voltages to their nominal values than with the default droop control.

Advances in reinforcement learning and deep learning have inspired application of these domains into electric power systems, and a review of recent and past research is presented in [10]. To specifically tackle the Volt-VAR problem, distributed Q-learning is adopted in [11] where the state and action space is discretized, 
and a state-action value table is updated in the learning process. In [12], deep learning is used for reactive power compensation, in a two-timescale representation of control, one for capacitor configuration and the other for inverter optimization.

Similar work on two-time-scale representation is presented in [13], where the objective is to minimize total line losses. In reference [13], the voltage regulation problem is reformulated to be solved by an equivalent semidefinite programming problem (SDP). It is shown that over a radial network, the algorithm applies to a decentralized setting and it is robust to communication failure. In our paper, we do not propose to minimize line losses but we do not require any communication at any time.

Most of the available methods on Volt-VAR control are based on steady state approximations of a radial network, and those which claim decentralized control still rely on communication at least between 1-hop-away neighbors. It is highlighted in [14] that cybersecurity issues in distribution grids with PV are not much explored, especially the effect of data manipulation.

Our main contribution in this paper is two-fold. First, we employ a quasi-steady state representation of how incremental changes in reactive power injection/consumption affect nodal voltages. This enables us to represent the network as a linear discrete-time system. We use the same linearized power flow model as the one assumed and extensively used in the body of literature. It should be noted that this linearized model is indeed valid across a wide range of operating conditions. Second, we provide a scheme for communication-free control, in which all measurements and actions are taken locally at the same node (i.e. 0-hop-away and no communication). We prove when this scheme works and when it fails by providing a closed-form representation of the set of control policies that can achieve stability in the sense of Lyapunov.

The rest of this paper is organized as follows. In Section 2, the problem of voltage regulation by reactive power compensation is formulated. Section 3 details one of our main contributions, the guarantees on stability and where the communication-free scheme fails. In Section 4 we propose a way to select control policies at every node from the region of feasible policies, and introduce a risk factor to do so. In Section 5, the theory is tested on a 56-bus distribution network where there is PV penetration at all nodes, and in Section 6 we provide concluding remarks.

\section{Problem formulation}

In this section, we introduce a commonly used power flow model for radial networks, we present our system in the form of a quasi-steady state discrete-time dynamical system, and we state the control objective. Although a linearized model is assumed in this section (Eq. (3)), we conduct simulations in Section 5 on the full non-linearized $\mathrm{AC}$ model of a radial distribution network (Eq. (1)).

\subsection{Linearized power flow model for radial networks}

A three-phase balanced radial network is assumed in this model of a distribution grid, with a single substation acting as the slack bus. The following formulation is proposed and used in [3-9, 12-15].

Let $\mathcal{N}=\{0,1,2, \ldots, n\}$ be the set of nodes (buses), with 0 representing the substation node (slack bus), and $\mathcal{L} \subset \mathcal{N} \times \mathcal{N}$ be the set of lines connecting these nodes. That is, for each pair of nodes, $i, j \in \mathcal{N}$, there is a line connecting them iff $(i, j) \in \mathcal{L}$. As a convention, let $(i, i) \notin \mathcal{L} \forall i \in \mathcal{N}$. The complex impedance of line $(i, j)$ is given by $z_{i j}=r_{i j}+\mathbf{i} x_{i j}$, where $\mathbf{i}=\sqrt{-1}$. The complex current and complex power flowing from node $i$ to node $j$ are given respectively as $I_{i j}$ and $S_{i j}=$ $P_{i j}+\mathbf{i} Q_{i j}$. The complex voltage and the complex power injection at node $i$ are given respectively as $V_{i}$ and $s_{i}=$ $p_{i}+\mathbf{i} q_{i}$. Furthermore, let $\mathcal{K}_{j, i} \subset \mathcal{N}$ be the set of all nodes connected directly to node $j$, excluding node $i$ (and $j$ ). That is, $\mathcal{K}_{j, i}=\{k \mid(j, k) \in \mathcal{L}, k \neq i\}$.

Therefore, the power flow equations are derived as follows:

$$
\begin{aligned}
P_{i j}= & \sum_{k \in \mathcal{K}_{j, i}} P_{j k}+r_{i j}\left|I_{i j}\right|^{2}-p_{j} & \forall(i, j) \in \mathcal{L} \text { (1a) } \\
Q_{i j}= & \sum_{k \in \mathcal{K}_{j, i}} Q_{j k}+x_{i j}\left|I_{i j}\right|^{2}-q_{j} & \forall(i, j) \in \mathcal{L} \text { (1b) } \\
\left|V_{j}\right|^{2}= & \left|V_{i}\right|^{2}-2\left(r_{i j} P_{i j}+x_{i j} Q_{i j}\right) & \\
& +\left(r_{i j}^{2}+x_{i j}^{2}\right)\left|I_{i j}\right|^{2} & \forall(i, j) \in \mathcal{L} \text { (1c) } \\
\left|I_{i j}\right|^{2}= & \frac{P_{i j}^{2}+Q_{i j}^{2}}{\left|V_{i}\right|^{2}} & \forall(i, j) \in \mathcal{L}(1 \mathrm{~d})
\end{aligned}
$$

For clarity, Eq. (1a) is depicted in Fig. 1. A linearization of Eq. (1), known as Simplified Distflow [4], is to approximate the squared current magnitudes, $\left|I_{i j}\right|^{2}$, as zero, implying that line losses are negligible compared to power flowing on the lines. While this assumption is used in the theoretical derivation, the simulations we present in this paper include more 
realistic lossy line scenarios $\left(\left|I_{i j}\right|^{2} \neq 0\right)$. Setting $\left|I_{i j}\right|^{2}=0 \forall(i, j) \in \mathcal{L}$ yields Eq. (2) in which changes in power $(p$ or $q)$ have a linear impact on changes in squared voltage.

$$
\begin{aligned}
P_{i j} & =\sum_{k \in \mathcal{K}_{j, i}} P_{j k}-p_{j} & \forall(i, j) & \in \mathcal{L} \\
Q_{i j} & =\sum_{k \in \mathcal{K}_{j, i}} Q_{j k}-q_{j} & \forall(i, j) & \in \mathcal{L} \\
\left|V_{j}\right|^{2} & =\left|V_{i}\right|^{2}-2\left(r_{i j} P_{i j}+x_{i j} Q_{i j}\right) & \forall(i, j) & \in \mathcal{L}
\end{aligned}
$$

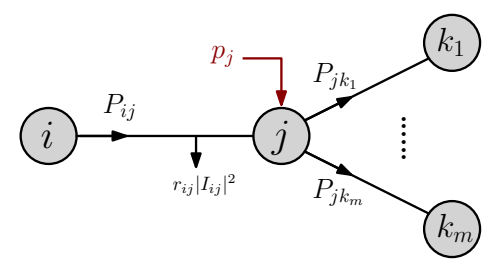

Figure 1. Depiction of Eq. (1a). Local power injection, $p_{j}$. Node $i$ is unique 'parent' to node $j$.

Let $v_{i}:=\left|V_{i}\right|^{2}$, and define the following vectors: $\mathbf{v}=\left[v_{1}, v_{2}, \ldots, v_{n}\right]^{T}, \mathbf{v}_{\mathbf{0}}=\left[v_{0}, v_{0}, \ldots, v_{0}\right]^{T}, \mathbf{p}=$ $\left[p_{1}, p_{2}, \ldots, p_{n}\right]^{T}$, and $\mathbf{q}=\left[q_{1}, q_{2}, \ldots, q_{n}\right]^{T}$. Since the network is radial, each node has only one parent node (connecting back to the substation) and hence a unique path to node 0 . It can be shown by induction that

$$
\mathbf{v}=R \mathbf{p}+X \mathbf{q}+\mathbf{v}_{\mathbf{0}}
$$

where the entries of matrices $R$ and $X$ at $i^{\text {th }}$ row and $j^{\text {th }}$ column are given in Eq. (4)

$$
\begin{aligned}
& R_{i j}:=2 \sum_{(h, k) \in \mathcal{P}_{i} \cap \mathcal{P}_{j}} r_{h k} \\
& X_{i j}:=2 \sum_{(h, k) \in \mathcal{P}_{i} \cap \mathcal{P}_{j}} x_{h k}
\end{aligned}
$$

and $\mathcal{P}_{i} \subset \mathcal{L}$ is the unique set of lines (or "path") connecting node $i$ all the way back to node 0 . That is, if we call $\mathcal{P}_{i}$ and $\mathcal{P}_{j}$ the set of 'ancestor nodes' to nodes $i$ and $j$ respectively, then $\mathcal{P}_{i} \cap \mathcal{P}_{j}$ is the set of common ancestor nodes. From this, it is evident that $X_{i j}=X_{k k}$ where node $k$ is the first (or 'youngest') common ancestor of nodes $i$ and $j$. This is illustrated in Fig. 2.

It can be seen also in Fig. 2 that even if two nodes are far apart (top sub-figure), they can still share many ancestors in common, as opposed to what is seen in the bottom sub-figure. $X_{k k}$ is larger for the former than it is

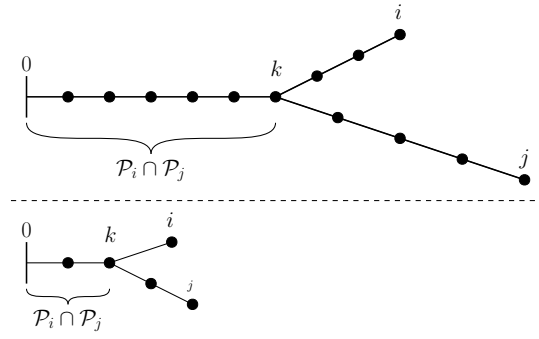

Figure 2. Illustration of $\mathcal{P}_{i} \cap \mathcal{P}_{j}$ : nodes $i$ and $j$ share many (top) vs. a few (bottom) ancestor nodes.

for the latter, thus $X_{i j}$ is also as such, even though they seem closer in the bottom one.

We compare the size of $X_{i j}$ for different pairs of nodes $i$ and $j$ to highlight the fact that injecting/consuming reactive power at node $i$ will have a higher impact on the voltage at node $j$ if they share more ancestor nodes than if they share less. This is justified in Eq. ( 3 \& 4).

What is also implied by Eq. (4) is that neighboring nodes have a stronger effect on one another the deeper they are into the network. From an intuitive point of view, we know that in distribution grids, voltage tends to sag more for nodes farther away from the substation. This aligns well with the properties of the linearized model given in Eq. ( 3 \& 4) and illustrated in Fig. 2.

\subsection{Quasi-steady state dynamical system}

Let $\mathbf{q}=\mathbf{q}^{\mathrm{PV}}+\mathbf{q}^{\text {other }}$ and $\mathbf{p}=\mathbf{p}^{\mathrm{PV}}+\mathbf{p}^{\text {other }}$. To isolate the effect of $\mathrm{PV}$ penetration and inverter control on the nodal voltages, define $\mathbf{v}^{\text {other }}:=R \mathbf{p}^{\text {other }}+X \mathbf{q}^{\text {other }}+\mathbf{v}_{\mathbf{0}}$. Eq. (3) can thus be rephrased as

$$
\mathbf{v}=R \mathbf{p}^{\mathrm{PV}}+X \mathbf{q}^{\mathrm{PV}}+\mathbf{v}^{\text {other }}
$$

Here, $\mathbf{p}^{\mathrm{PV}}$ is the vector of active powers injected/provided by the PV modules at all nodes. On the other hand, $\mathbf{q}^{\mathrm{PV}}$ is the vector of reactive powers injected by the PV inverters at all nodes, and it is the control input in this paper. For generality, if there is no $\mathrm{PV}$ module at node $i$, then $p_{i}^{\mathrm{PV}}=q_{i}^{\mathrm{PV}}=0$.

Eq. (5) represents a steady-state algebraic relationship between nodal power injections and squared nodal voltages. However, if we define a discrete-time system with a sufficiently large time step, we may express the system of Eq. (5) as a quasi-steady-state system, where all the terms update at every discrete time step. That is, for every time step $t$, each PV unit at node $i$ injects $p_{i}^{\mathrm{PV}}(t)$ and $q_{i}^{\mathrm{PV}}(t)$, and the squared voltage at node $j$ settles at time step $t+1$ to $v_{i}(t+1)$. Thus, the following holds true:

$$
\mathbf{v}(t+1)=R \mathbf{p}^{\mathrm{PV}}(t)+X \mathbf{q}^{\mathrm{PV}}(t)+\mathbf{v}^{\text {other }}(t)
$$


from which Eq. (7) below can be derived as a discrete-time dynamical equation which captures incremental updates in nodal voltages. Please note that we assume the PV inverters are capable of switching fast enough such that changes in the real power injected by the PV modules does not change as quickly.

$$
\begin{aligned}
\mathbf{v}(t+1)=\mathbf{v}(t) & +R\left[\mathbf{p}^{\mathrm{PV}}(t)-\mathbf{p}^{\mathrm{PV}}(t-1)\right] \\
& +X\left[\mathbf{q}^{\mathrm{PV}}(t)-\mathbf{q}^{\mathrm{PV}}(t-1)\right] \\
& +\left[\mathbf{v}^{\text {other }}(t)-\mathbf{v}^{\text {other }}(t-1)\right]
\end{aligned}
$$

In the communication-free control proposed in this paper, we assume that at each node $i$, only $v_{i}(t)$ is observed and only $q_{i}(t)$ can be controlled, so from the perspective of the controller at node $i$, Eq. (7) is seen as:

$$
\begin{aligned}
v_{i}(t+1)=v_{i}(t) & +X\left[q_{i}^{\mathrm{PV}}(t)-q_{i}^{\mathrm{PV}}(t-1)\right] \\
& +\operatorname{unknown}_{i}(t)
\end{aligned}
$$

Note also that the difference terms of $\mathbf{p}^{\mathrm{PV}}$ and $\mathbf{v}^{\text {other }}$ in Eq. (7) might be approximated to zero in general in a small time-scale if the control time of $\mathbf{q}^{\mathrm{PV}}$ is relatively small. As an example, the power delivered due to sunlight would generally not change as quickly as the PV inverter's control time. This makes the unknown term in Eq. (8) relatively small.

\subsection{Inverter capacity model}

As per IEEE Standard 1547-2018 [16], each PV inverter is "capable at all times to absorb or inject reactive power, to the full extent of the reactive power capability ranges". These ranges are defined in the document, e.g. $44 \%$ of nameplate capacity.

As modelled in [1-3] and many more recent papers as well, the capacity of $q_{i}^{\mathrm{PV}}(t)$ is bounded in magnitude by $\sqrt{\left|s_{\max }\right|^{2}-\left(p_{i}^{\mathrm{PV}}(t)\right)^{2}}$ and this is depicted in Fig. 3. This means that if there is less sunlight, there is more reactive capacity available, with maximum inverter capacity $\left|s_{\max }\right|$.

\subsection{Objective formulation}

The objective of the local controller at each node $i$ is to minimize the magnitude of $e_{i}(t)$, where

$$
e_{i}(t):=1-v_{i}(t)
$$

is the local squared voltage deviation from the nominal value of 1 p.u..

To generalize for all controllers, and to formulate an objective statement, all that matters is voltage deviation

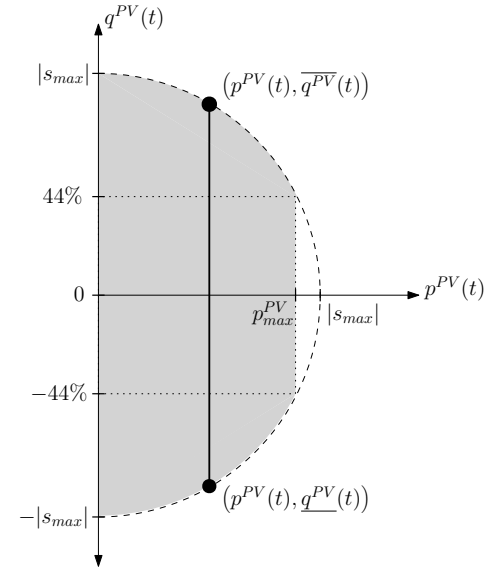

Figure 3. Illustration of reactive power capacity as a function of real power injected by PV.

from nominal value, since PV inverters can provide the reactive power compensation 'for free' up to a limit in magnitude. Therefore, penalizing the amount of reactive power injected/consumed is irrelevant. However, in attempt to minimize variations of reactive power flow changes across the network, we penalize changes in reactive power injection (i.e. $\left[q_{i}^{\mathrm{PV}}(t)-q_{i}^{\mathrm{PV}}(t-1)\right]$. This is desired to minimize line losses and uncertainty of the operating condition around which we assumed a linearized system model. In other words, to better track changes in the operating condition, we seek less fluctuation in reactive power compensation. The localized objective at node $i$ becomes:

$$
\begin{array}{ll}
\min _{q_{i}} & \sum_{t=0}^{\infty}\left[e_{i}(t)\right]^{2}+\mu_{i}\left[q_{i}^{\mathrm{PV}}(t)-q_{i}^{\mathrm{PV}}(t-1)\right]^{2} \\
\text { s.t. } & \underline{q_{i}^{\mathrm{PV}}}(t) \leq q_{i}^{\mathrm{PV}}(t) \leq \overline{q_{i}^{\mathrm{PV}}}(t)
\end{array}
$$

where $\mu_{i}$ is a weighting factor assigned for each node $i$ and $q_{i}^{\mathrm{PV}}(t)$ and $\overline{q_{i}^{\mathrm{PV}}}(t)$ are known at time $t$ but not controllable.

We propose a stationary linear control policy at each node in attempt to meet the objective of Eq. (10). Let $g_{i}$ be a feedback control gain, determined for each node $i$ by prescribed setting. The control input, $q_{i}^{\mathrm{PV}}(t)$, is updated incrementally at every point in time as a scalar multiple of tracking error $e_{i}(t)$ (refer to Eq. (9)), as 
shown in Eq. (11a).

$$
\begin{aligned}
q_{i}^{\mathrm{PV}}(t) & =q_{i}^{\mathrm{PV}}(t-1)+g_{i} e_{i}(t) \\
\Rightarrow \mathbf{e}(t+1) & =(I-X G) \mathbf{e}(t) \\
& -R\left[\mathbf{p}^{\mathrm{PV}}(t)-\mathbf{p}^{\mathrm{PV}}(t-1)\right] \\
& -\left[\mathbf{v}^{\text {other }}(t)-\mathbf{v}^{\text {other }}(t-1)\right]
\end{aligned}
$$

where $G$ is a diagonal matrix whose $i^{\text {th }}$ entry on the diagonal is $g_{i}$. The fact that $G$ is diagonal, and the control policy shown in Eq. (11a) both illustrate the communication-free aspect of the proposed control scheme.

Eq. (11b) is a transformed version of Eq. (7). Please note that the application of Eq. (11a) (and hence Eq. (11b)) assumes no violation of the constraint in Eq. (10b). At any point in time, the controller is assigned to saturate at the capacity limit if the constraint is about to be violated. This ensures Eq. (10b). Furthermore, as previously explained, the difference terms of $\mathbf{p}^{\mathrm{PV}}$ and $\mathbf{v}^{\text {other }}$ in Eq. (11b) might be approximated to zero, as explained for Eq. (7). This is assumed for small time scales, but as we know, over a whole day, the $\mathbf{p}^{\mathrm{PV}}$ terms may heavily fluctuate. The controller considers this as disturbance and is able to reject it by feedback.

\section{Guarantees on stability under communication-free policies}

In this section, we show that there exists a convex set of possible matrices $G$, which we will call the stability region, such that the control scheme in Eq. (11) drives $e_{i}(t) \rightarrow 0$ for all nodes $i$. The fact that $G$ is diagonal emphasizes the communication-free aspect. We propose and prove a list of properties of this stability region and detail under what policies the system succeeds/fails to converge.

Definition 1 (Communication-free linear system). A system is labelled C.F. if it obeys the following discrete-time dynamics

$$
\mathbf{e}(t+1)=(I-X G) \mathbf{e}(t)
$$

where $X$ is real, symmetric and positive-definite, $G$ is diagonal and $\mathbf{e}(t) \in \mathbb{R}^{n}$. The notation C.F. $\Omega$ is used to indicate a system under which all nodes are still represented, but only those in $\Omega$ are active, i.e. strictly $G_{i i}=0 \forall i \neq \Omega$.

Remark 1.1. Please refer to the Nomenclature section. Eq. (12) implies Eq. (13) under C.F. $\Omega$.

$$
\mathbf{e}_{\boldsymbol{\Omega}}(t+1)=\left(I-X_{\boldsymbol{\Omega}} G_{\boldsymbol{\Omega}}\right) \mathbf{e}_{\boldsymbol{\Omega}}(t)
$$

That is, if all nodes outside of $\boldsymbol{\Omega}$ are inactive, then from the perspective of the controllers at nodes $\Omega$ where regulation of only those nodes' voltages is of interest, the larger system C.F. becomes equivalent to the local subsystem C.F. $\Omega$. That is not to say that the other nodes won't get affected by actions of nodes in $\Omega$, but it means that nodes in $\Omega$ are not affected by actions at nodes not in $\Omega$, simply because they are necessarily inactive.

Remark 1.2. From Eq. (11a), we may deduce that if during any time period, $q_{i}^{\mathrm{PV}}(t)$ at some node is saturated at its limit, then the corresponding $g_{i}$ value is effectively zero (no change in reactive power injection). This is equivalent to node $i$ becoming inactive during that period.

Definition 2 (Stability). We say C.F. $\Omega$ is stable iff

$$
\rho\left(I-X_{\boldsymbol{\Omega}} G_{\boldsymbol{\Omega}}\right)<1
$$

where $\rho(A)$ is the spectral radius of some matrix $A$.

Remark 2.1. This definition implies that whether $e_{i}(t)$ diverges or not for each $i \notin \Omega$ is irrelevant, instead, all what matters is the voltage stability at nodes in $\Omega$.

Definition 3 (Sub-matrix). " $G_{\Psi} \sqsubset G_{\boldsymbol{\Omega}}$ " is a logical statement that is true iff for each $i \in \Psi \subset \boldsymbol{\Psi}$, the entry corresponding to node $i$ in $G_{\boldsymbol{\Psi}}$ is identical to that in $G_{\boldsymbol{\Omega}}$. Remark 3.1. Given some $G_{\boldsymbol{\Omega}}$ and any $\boldsymbol{\Psi} \subset \boldsymbol{\Omega}$, there is a unique $G_{\Psi}$ such that $G_{\Psi} \sqsubset G_{\boldsymbol{\Omega}}$.

Using the definitions above, we propose multiple theorems below and provide the proofs in the appendix in reference [17]. Please note that these theorems can be applied to linear systems beyond the scope of this paper, as long as they satisfy Definition 1.

Theorem 1 (Slower policies stable). If C.F. $\Omega$ is stable under some control policy $G_{\Omega}$, then it is also stable under $\tilde{G}_{\boldsymbol{\Omega}}:=G_{\boldsymbol{\Omega}} \Theta$, where $\Theta$ is diagonal and each entry on the diagonal lies in $(0,1]$.

Since we are considering a communication-free control scheme, it is not available information at each node what the next move at the other nodes will be. As a reminder, there is a control policy gain (scalar) $g_{i}$ at each node $i$ that dictates the local control scheme. Say under some combination of these policies, the system is already stable. In this scenario, it is worth asking how the changes to these policies at some nodes will affect other nodes. Theorem 1 states that if we begin with a combination of $g_{i}$ 's that stabilizes the system, then if any or multiple of those values is/are reduced in magnitude (i.e. 'slower'), the entire system will remain stable.

This is key in building a communication-free setting since it helps the controllers at all nodes know that if 
other nodes are less aggressive, perhaps due to scarcity of resources, then if their own policy remains put, the local voltage will not diverge.

Theorem 2 (Sub-system also stable). For any $\boldsymbol{\Psi} \subset \boldsymbol{\Omega}$, if C.F. $\Omega$ is stable under some control policy $G_{\boldsymbol{\Omega}}$, then C.F. $\Psi$ is stable under $G_{\Psi}$, where $G_{\Psi} \sqsubset G_{\boldsymbol{\Omega}}$.

Corollary 2.1 (Self-interest necessary). A necessary condition for C.F. $\Omega$ to be stable under some control policy $G_{\boldsymbol{\Omega}}$ is that for each $i \in \boldsymbol{\Omega}$, C.F. ${ }_{\{i\}}$ is stable under $g_{i}$, where $g_{i} \sqsubset G_{\Omega}$.

Corollary 2.2 (All-active sufficient). For any $\boldsymbol{\Psi} \subset \boldsymbol{\Omega}$ where $|\boldsymbol{\Omega}|=n$, a sufficient condition for C.F. $\Psi$ to be stable is that C.F. $\Omega$ is stable for the same set of policies.

It is worth clarifying the difference between Theorem 1 and Theorem 2. The first theorem is concerned with the stability at all nodes in $\Omega$; whereas the second is concerned with that of only a few nodes, $\boldsymbol{\Psi}$. That is if nodes in $\boldsymbol{\Omega}$ outside of $\boldsymbol{\Psi}$ became inactive, although stability at those nodes may be compromised, stability at $\boldsymbol{\Psi}$ will not. This is another key in establishing a communication-free scheme, as it guarantees that sudden absence or interruption of control in other parts of the distribution grid will not lead to local instability - assuming the system was stable prior to the interruption. Thus, knowledge of such remote interruptions is not required to ensure local stability.

Corollaries 2.1 and 2.2 consider application of Theorem 2 for scenarios with a single node active and all nodes active respectively. They are logical implications of the theorem, yet they are individually very useful in establishing and testing choice of control policies.

Corollary 2.1 suggests that it is necessary that each node seeks self-interest, otherwise no node will achieve stability. 1) This justifies the notion of self-interest in a communication-free setting, and 2) we may easily compute necessary bounds on any single node's control policy $g_{i}$ by hand (see Theorem 3) since the system C.F. $\{i\}$ is comprised of only one active node.

Corollary 2.2 suggests that if there is a feasible combination of $g_{i}$ 's that we know can stabilize the system, then by picking out any subset of those and setting the rest to zero, we may guarantee stability for the subset. This is tremendously useful in addressing the curse of dimensionality. As an example, consider the network in the case study (Section 5). Since $n=55$, there is a total of $3.6 \times 10^{16}$ possible combinations of subsystems to consider. Instead, by simulating offline over 1 only (where all nodes are active), we may achieve a good starting point for what the $g_{i}$ at each node should be for any subsystem of the 55 nodes.
Theorems 1 and 2 provide properties of the stability region over the space of possible policies, yet they do not present how the region looks like, and how it is bounded along more than a single dimension. Theorem 3 depicts this region more concretely.

Theorem 3 (Stability Region). For any system C.F. $\Omega$, let $\mathrm{g}_{\Omega}$ be a point in $\mathbb{R}^{\mid \boldsymbol{}}$, and each coordinate $g_{i}$ be the control policy at node $i \in \Omega$, so that $G_{\Omega}=\operatorname{diag}\left(\mathrm{g}_{\Omega}\right)$.

1. The control policy stability region is the set of all $\mathbf{g}_{\boldsymbol{\Omega}}$ such that both $G_{\boldsymbol{\Omega}}$ and $\left(2\left(X_{\boldsymbol{\Omega}}\right)^{-1}-G_{\boldsymbol{\Omega}}\right)$ are positive definite (i.e. $0 \prec G_{\Omega} \prec 2\left(X_{\Omega}\right)^{-1}$ ). Note: $\left(X_{\boldsymbol{\Omega}}\right)^{-1} \neq\left(X^{-1}\right)_{\boldsymbol{\Omega}}$.

2. This region is convex.

3. It is contained entirely in the open box $\mathcal{B}_{\boldsymbol{\Omega}}:=\left\{\mathbf{g}_{\boldsymbol{\Omega}} \mid X_{i i} g_{i} \in(0,2) \forall i \in \boldsymbol{\Omega}\right\} \subset \mathbb{R}_{+}^{|\boldsymbol{\Omega}|}$ (all-positive orthant of $\mathbb{R}^{|\Omega|}$ ).

4. The entire region is the union of all open segments bounded on one end by the origin and on the other end by the manifold of points $\mathbf{g} \in \mathcal{B}_{\Omega}$ that satisfy $f_{\boldsymbol{\Omega}}(2)=0$, where $f_{\boldsymbol{\Omega}}(\cdot)$ is defined as follows:

$$
\begin{aligned}
& f_{\boldsymbol{\Omega}}(\gamma):=\operatorname{det}\left(\gamma I-X_{\boldsymbol{\Omega}} G_{\boldsymbol{\Omega}}\right) \\
& =\sum_{k=0}^{|\boldsymbol{\Omega}|}(-1)^{k}\left[\sum_{\substack{\boldsymbol{\Psi} \subseteq \boldsymbol{\Omega} \\
|\boldsymbol{\Psi}|=k}} \operatorname{det}\left(X_{\boldsymbol{\Psi}}\right) \prod_{i \in \boldsymbol{\Psi}} g_{i}\right] \gamma^{|\boldsymbol{\Omega}|-k}
\end{aligned}
$$

Note: The determinant of an empty matrix is 1.

Corollary 3.1 (Multilinear mapping). For any $\gamma, f_{\boldsymbol{\Omega}}(\gamma)$ is multi-affine in $\mathrm{g}_{\Omega}$. That is for any $i \in \Omega$, define $\boldsymbol{\Psi}$ such that $\boldsymbol{\Omega}=\boldsymbol{\Psi} \cup\{i\}$ and $i \notin \boldsymbol{\Psi}$, then $f_{\boldsymbol{\Omega}}(\gamma)$ is affine in $g_{i}$. Namely, there is a polynomial in $\gamma, h_{\Psi}(\gamma)$, independent of $g_{i}$ such that

$$
f_{\boldsymbol{\Omega}}(\gamma)=\gamma \cdot f_{\boldsymbol{\Psi}}(\gamma)+g_{i} \cdot h_{\boldsymbol{\Psi}}(\gamma) \quad \forall \gamma
$$

Corollary 3.2 (Nested stability regions). The stability region of C.F. $\Psi$ is the projection of the stability region of C.F. $\Omega$ onto $\mathbb{R}^{|\boldsymbol{\Psi}|}\left(g_{i} \rightarrow 0 \forall i \notin \boldsymbol{\Psi}\right)$, for any $\boldsymbol{\Psi} \subset \boldsymbol{\Omega}$.

Corollary 3.3 (Simplex stable). All points inside the simplex $\left\{\begin{array}{l|l}\mathrm{g}_{\boldsymbol{\Omega}} \in \mathcal{B}_{\boldsymbol{\Omega}} & \sum_{i \in \boldsymbol{\Omega}} X_{i i} g_{i} \leq 2\end{array}\right\}$ are guaranteed to be inside the stability region of C.F. $\Omega$. 
Theorem 3 provides the requirement that all $g_{i}$ 's must be strictly positive to ensure local stability. That is, drop in voltage requires injection of reactive power, and increase requires consumption, following power systems experience. The convexity of the region implies that if a learning algorithm has a history of multiple stabilizing policies, then it knows that any convex combination of those policies is guaranteed to be stable. The open box provides strict upper and lower bounds. Finally, the effect of each individual node's policy $g_{i}$ on the stability of the whole system is highlighted, and yields results in the corollaries that follow.

Corollary 3.1 addresses the effect of each $g_{i}$ on the quantity $f_{\boldsymbol{\Omega}}(\gamma)$. Per the theorem, the algebraic equation in terms of $\mathbf{g}_{\boldsymbol{\Omega}}\left(f_{\boldsymbol{\Omega}}(2)=0\right)$ represents the stability region's (outer) boundary. For some fixed node $i$, changes in $f_{\boldsymbol{\Omega}}(2)$ are linear in changes in $g_{i}$. Moreover, if the policies are fixed at all nodes in $\Psi$ (where $\boldsymbol{\Omega}=\boldsymbol{\Psi} \cup\{i\}$ ), the policy for $i$ at the boundary of the stability region can be found directly by Eq. (16) as $-2 f_{\boldsymbol{\Psi}}(2) / h_{\boldsymbol{\Psi}}(2)$.

As an example, consider a system with 2 nodes active $(\boldsymbol{\Omega}=\{1,2\})$, and $X_{11}=1, X_{22}=2, X_{12}=$ $X_{21}=0.5$. It is illustrated in Fig. 4 that if $g_{2}$ is fixed at 0.6 , then the most aggressive the policy at node 1 can be is roughly 1.68 (red point), which was found by $-2 f_{\boldsymbol{\Psi}}(2) / h_{\boldsymbol{\Psi}}(2) \approx 1.68$ for $\boldsymbol{\Psi}=\{2\}$. In a sense, there is a total amount of energy that is to be provided, so if one node provides less, the other can provide more.

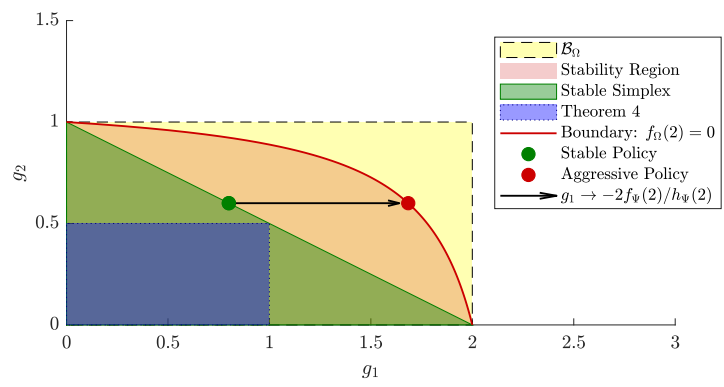

Figure 4. Possible regions of C.F. systems. Small box of Theorem 4 (blue) $\subset$ Simplex (green) $\subset$ Stability Region (red) $\subset$ open box $\mathcal{B}_{\Omega}$ (yellow). Here, $\boldsymbol{\Psi}=\{2\}$.

Corollary 3.2 and 3.3 can be verified by Fig. 4 . Project the red region onto the horizontal axis, and indeed it will yield the region $\left(0,2 / X_{11}\right)$, the stability region of C.F. $\{1\}$. The green region is the simplex mentioned in the corollary.

Theorem 4 (Node-Invariant Policy). For any C.F. $\Omega$, if each node $i \in \boldsymbol{\Omega}$ adopts any policy $g_{i} \in\left(0,2 / m X_{i i}\right)$, independent of other nodes, where $m=|\boldsymbol{\Omega}|$, then C.F. $\Omega$ is guaranteed to be stable.
Theorem 4 says that there is a small box of policies (see Fig. 4) which is guaranteed to be inside the stability region. The fact that it is a box implies that for any group of nodes, if the policies are fixed and the system is stable, then the other nodes can roam around inside the box freely and the system will remain stable. Because the box is much smaller for higher order system, it is considered as too conservative, yet the tip of the box (farthest from origin) may be considered a good starting point for a searching algorithm. The tip of this box is addressed in Section 4.

Please note that the illustration shown in Fig. 4 does not apply only to the given example. For any radial network, of any size, which can be reasonably modelled as a C.F. system (Eq. (12)), there will always be an outer box (yellow), an inner box (blue), and a simplex (green), and the boundary of the stability region (red) will be always be between the simplex and the outer box!

\section{Choice of stationary policies from stability region}

The previously mentioned theorems and corollaries establish bounds on the choice of policy $g_{i}$ for every node $i$, but the question remains: where inside the stability region should the controllers operate? In this section, we propose a control schemes which is based on policies that are fixed in time, i.e. stationary policies, to answer the question.

The objective of Eq. (10) is based on a single-node selfish objective. Since each node is unaware of the presence or absence of other activity in the network, the unknown term of Eq. (8) is assumed by default to follow a white noise behavior, thus the objective becomes an LQG control objective. It involves solving a Ricatti equation, whose solution yields a stationary policy of

$$
g_{i}^{*}=\frac{1}{X_{i i}} \frac{2}{1+\sqrt{1+\frac{4 \mu_{i}}{X_{i i}^{2}}}} \forall i
$$

where $\mu_{i}$ is the control penalty factor in Eq. (10). The reason we can analytically express the optimal gain as in Eq. (17) is because the single-node scalar system can be solved by hand.

The factor which determines if this distribution of policies $g_{i}^{*} \forall i$ will yield stability or not is the choice of $\mu_{i} \forall i$. This value needs to be preset for each node. Considering we desire to dispatch this algorithm to all nodes, we would like a strategy that is as unbiased towards any node as possible. To do this, we manipulate 
Eq. (17). For some $\theta_{i} \in(0,1)$, assign $\mu_{i}$ as:

$$
\begin{aligned}
\mu_{i} & \leftarrow \frac{X_{i i}^{2}}{4}\left[\left(\frac{1}{\theta_{i}}-1\right)^{2}-1\right] \\
\Rightarrow g_{i}^{*} & \leftarrow \theta_{i} \frac{2}{X_{i i}}
\end{aligned}
$$

As shown in Theorem 3, the open box places upper and lower bounds which require $g_{i} \in\left(0,2 / X_{i i}\right)$. By the trick used in Eq. (18b), we define necessary conditions of $\theta_{i} \in(0,1) \forall i$ to remain inside the box, and sufficient conditions of $\theta_{i} \in(0,2 / m) \forall i$ for a guarantee of stability ( $m$ is the total number of active nodes with PV) is provided by Theorem 4 .

The only remaining task is to pick out $\theta_{i}$, and one way to do it is $\theta_{i} \leftarrow$ constant $\forall i$, where one may perform offline search over the best constant (i.e. node-unbiased). Although $\theta_{i}$ may seem unbiased over the set of nodes, keep in mind that $g_{i}^{*}$ is still influenced by $X_{i i}$ as shown in Eq. (18b). This is justified by the fact that nodes deeper into the network have a higher impact on the grid (see Eq. (4)) and must be set to follow more conservative policies. This is exactly what is being proposed in Eq. (18b) for constant $\theta_{i}$.

From Eq. (18b), one may realize $\theta_{i}$ as a risk factor, where $\theta_{i}=1 / m$ is considered as risk-balanced, $\theta_{i} \gg 1 / m$ as heavy risk-seeking or too strong/aggressive, and $\theta_{i} \ll 1 / m$ as very risk-averse or too weak/safe/conservative. A case study of this is performed in Section 5. See Fig. 8 and Fig. 9. In those results, we assume that the same value of $\theta$ is dispatched to all nodes (i.e. all are equally risky). However, it is also possible that at individual nodes, a controller free-rides the local $\theta$ value by tuning it arbitrarily within $(0,1)$, perhaps to save energy locally if other buses are already providing enough reactive power. For now, this is left to future work, where a learning scheme is adopted at each node to find the best local $\theta$.

This risk terminology is justified by Fig. 4. It can be seen that the weaker the policy is at one node, the more expanded the stability region will become for the other nodes. That is, if any group of nodes become more risk-averse, the nodes everywhere else get to be more risk-seeking without escaping the stability region, and vice-versa. In general, to dispatch an unbiased set of $\theta_{i}$ 's, we assume risk-balanced policies at all nodes as a safe bet, i.e. $\theta_{i} \approx 1 / m \forall i$.

It is worth noting though that $m$ is the number or active PV nodes, not the total number of nodes in the system. This means that in a system with many nodes, $1 / m$ is small only when many PV's are actively engaged in reactive power compensation. In general, that is not bad because the more the better, since the injection of power by one is received by the other. It is reasonable to then expect that, for stability purposes, the bounds on each injection is inversely proportional to the number of active nodes, which is precisely captured in the $1 / \mathrm{m}$ factor, presented here and in Theorem 4.

\section{Case study}

We test our theory on a 56-bus Southern California Edison distribution grid (12 kV, 1 MVA base) as used in [7]. A schematic of the network is shown in Fig. 5. We modified the case file for this network developed by [15] to accompany MATPOWER [18], which we used in MATLAB for our simulations. For example, we changed all $P V$-type buses ( $P V$ here in the power flow sense, not photovoltaic) to $P Q$-type, i.e. all are $P Q$, except the slack.

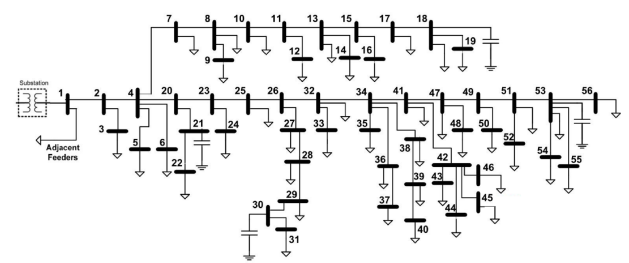

Figure 5. Schematic of 56-bus distribution grid [7].

Moving forward, we will return to use "PV" to mean photovoltaic. We assume PV penetration at all nodes other than the slack bus, along with the possibility of reactive power injection at all buses with PV. There is a total of $n=55$ possible active nodes (since bus 1 is slack). Another modification we made was to remove all sources of steady state active and reactive power injection, and replace them with our own, as follows. Shown in Fig. 6 (top), PV penetration was taken from real data obtained publicly thanks to Pecan Street's Dataport website [19]. We multiply the 24-hour PV profile by 200 to suggest that at each node, there are that many homes contributing a relatively similar profile.

The load profile shown in Fig. 6 (bottom) is not obtained from real data, but is modelled in such a way that the sum of the loads from various nodes does not simply cancel out. This allows for testing the proposed control scheme under harsher loading conditions.

\subsection{Voltage performance with no control}

Under the real power injection and consumption shown in Fig. 6, and zero control, i.e. no reactive power compensation, we obtain the results shown in Fig. 7. The voltage is too low when the system is heavily loaded 

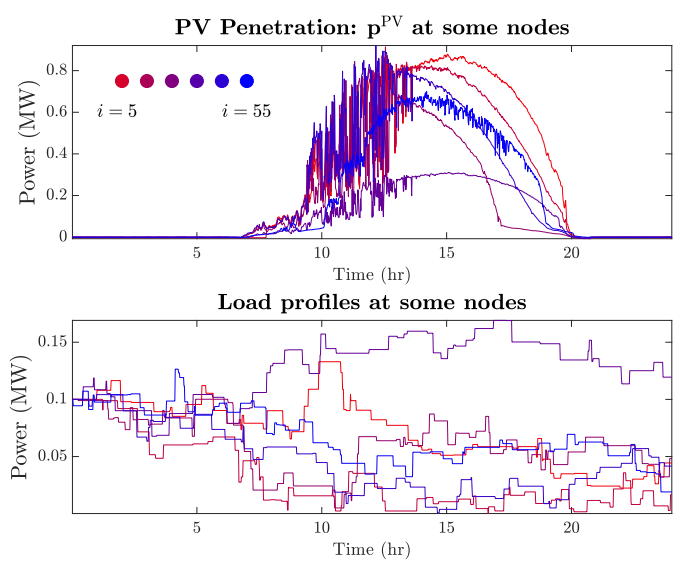

Figure 6. PV penetration based on real data [19], and randomized load patterns. Each color is a node.

and too high during peak PV penetration. In addition, the cumulative cost curve in Fig. 7 (right) shows steady increase where the voltage is far from desired. The cost curve is based on the objective in Eq. 10. It is unitless, and to be compared with the curves in Fig. 8 \& 9 .
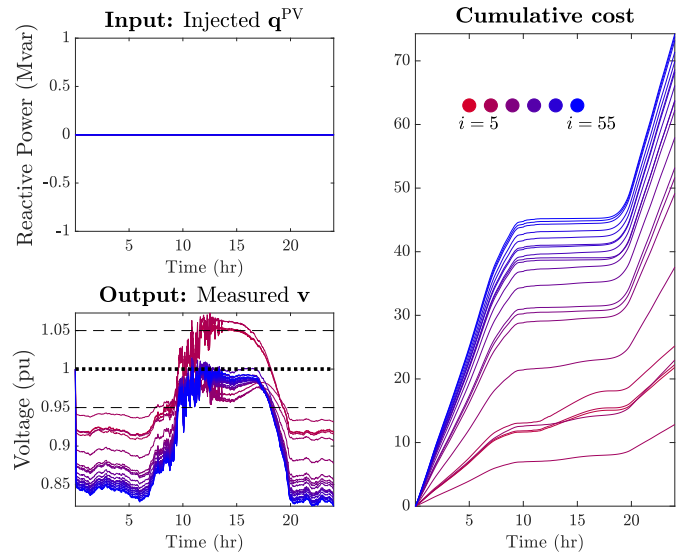

Figure 7. No control whatsoever. Over-voltage during peak sunlight and under-voltage due to heavy loading elsewhere. Cumulative cost: see Eq. (10).

\subsection{Stationary communication-free policy}

Based on Theorems 3 and 4, we adopt a stationary policy for each node that we know will ensure stability. That is, at all times, each controller simply measures the squared voltage deviation from 1 p.u., multiplies it by $g_{i}$ (constant over the 24 hours), and determines the reactive power compensation needed by the PV inverter.

We follow what was presented in Eq. (18b) to select the appropriate $g_{i}$. We select a risk-balanced (see Section 4) value of $\theta_{i}=1 / 55$ since $m=55$, then modify this value by adding a relatively smaller random number. That is, we are still somewhere inside the stability region, as guaranteed by the Theorem 4 , but the results in Fig. 8 show that even with small additive randomness in choice of policy, we achieve desirable results. The figure corresponds to a scenario with the similar loading and PV penetration conditions depicted in Fig. 7, and the aforementioned control strategy is initiated at $5 \mathrm{AM}$.

It can be observed that the cost curve flattens almost immediately after control is applied, and it increases noticeably only around heavy peak PV penetration times, around early afternoon.
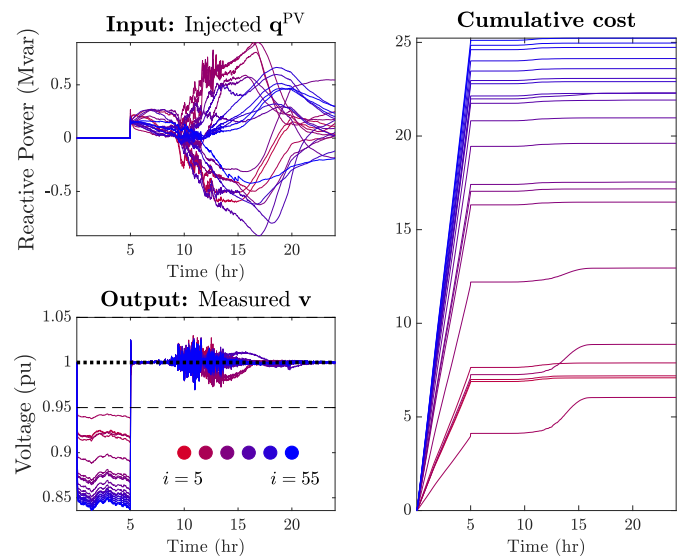

Figure 8. Stationary control with risk-balanced policy $\left(\theta_{i} \approx 1 / m\right)$. Voltage is bounded \& cumulative cost settles.

We then consider the same scenario but with a much weaker policy, namely with much smaller magnitude for each $g_{i}$. This is done by selecting a very risk-averse or over-conservative $\theta_{i} \approx 0.01 / \mathrm{m}$. Results are shown in Fig. 9, and prove that if controllers are too weak, they can be overwhelmed by deep PV penetration, and need to be more aggressive.

\section{Concluding remarks}

In this paper, we consider the Volt-VAR problem in distribution networks with deep PV penetration. We reformulated a commonly used power flow model as a quasi-steady state discrete-time dynamical system to directly relate incremental changes in reactive power to incremental changes in voltage (Eq. (7)). We use this to show that if the controller at each node adopts a stationary linear feedback policy by only measuring and reacting to voltage at the same node, the entire system can achieve desired voltage levels. 

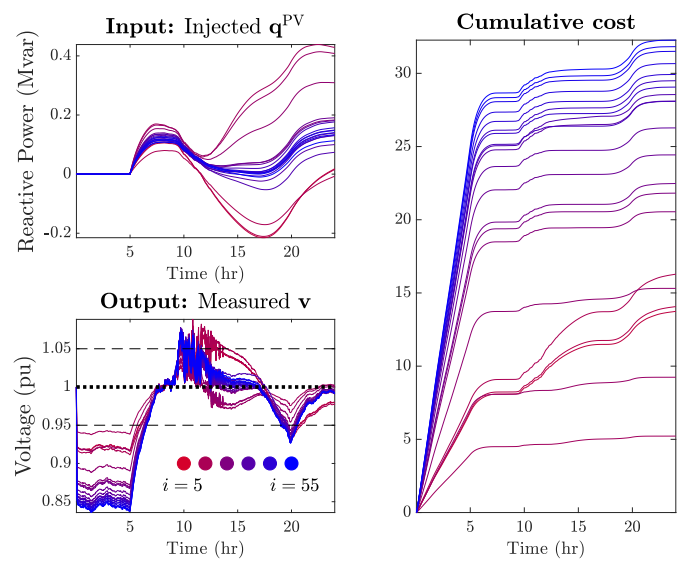

Figure 9. Stationary control with weak policy $\left(\theta_{i} \approx 0.01 / m\right)$. Unstuitable with deep PV penetration.

This implies that a communication-free distributed control scheme which stabilizes the system by reactive power compensation is possible. Our main contribution lies in providing an exact and closed form depiction of the set of feedback gains (control policies) that can make this possible. That is, given a set of line parameters for any radial network, using our result, the reader may quickly derive upper and lower bounds on the control policies for a communication-free setting.

As part of ongoing and future work, we consider the scenario where the line parameters are not even provided to the user, and the controller at each node should determine what policy to adopt. This presents an opportunity to use reinforcement learning and other learning-based methods to actively learn from past local measurements what best actions to take.

\section{Acknowledgement}

This work is supported in part by NSF Grant ECCS-1839616 and ECCS-1611301. The first author would like to thank Mr. Xinbo Geng, Mr. Tong Huang and Dr. Bin Wang for their time and feedback on the derivation and verification of the model formulation and proof of theorems.

\section{References}

[1] E. Liu and J. Bebic, "Distribution system voltage performance analysis for high-penetration photovoltaics," tech. rep., Feb. 2008.

[2] K. Turitsyn, P. Sulc, S. Backhaus, and M. Chertkov, "Options for control of reactive power by distributed photovoltaic generators," Proceedings of the IEEE, vol. 99, pp. 1063-1073, June 2011.

[3] V. Kekatos, G. Wang, A. J. Conejo, and G. B. Giannakis, "Stochastic reactive power management in microgrids with renewables," IEEE Transactions on Power Systems, vol. 30, pp. 3386-3395, Nov. 2015.

[4] G. Qu and N. Li, "Optimal distributed feedback voltage control under limited reactive power," arXiv preprint arXiv:1810.11121v1, 2018.

[5] S. Magnússon, G. Qu, and N. Li, "Distributed voltage control with communication delays," arXiv preprint arXiv:1903.01065, 2019.

[6] S. Magnusson, G. Qu, C. Fischione, and N. Li, "Voltage control using limited communication," IEEE Transactions on Control of Network Systems, 2019.

[7] M. Farivar, R. Neal, C. Clarke, and S. Low, "Optimal inverter VAR control in distribution systems with high PV penetration," in 2012 IEEE Power and Energy Society General Meeting, IEEE, July 2012.

[8] W. Lin, R. Thomas, and E. Bitar, "Real-time voltage regulation in distribution systems via decentralized PV inverter control," in Proceedings of the 51st Hawaii International Conference on System Sciences, Hawaii International Conference on System Sciences, 2018.

[9] H. Zhu and H. J. Liu, "Fast local voltage control under limited reactive power: Optimality and stability analysis," IEEE Transactions on Power Systems, vol. 31, pp. 3794-3803, Sept. 2016.

[10] M. Glavic, R. Fonteneau, and D. Ernst, "Reinforcement learning for electric power system decision and control: Past considerations and perspectives," IFAC-PapersOnLine, vol. 50, pp. 6918-6927, July 2017.

[11] Y. Xu, W. Zhang, W. Liu, and F. Ferrese, "Multiagent-based reinforcement learning for optimal reactive power dispatch," IEEE Transactions on Systems, Man, and Cybernetics, Part C (Applications and Reviews), vol. 42, pp. 1742-1751, Nov. 2012.

[12] Q. Yang, G. Wang, A. Sadeghi, G. B. Giannakis, and J. Sun, "Real-time voltage control using deep reinforcement learning," 2019.

[13] B. Zhang, A. Y. Lam, A. D. Dominguez-Garcia, and D. Tse, "An optimal and distributed method for voltage regulation in power distribution systems," IEEE Transactions on Power Systems, vol. 30, pp. 1714-1726, July 2015.

[14] Y. Isozaki, S. Yoshizawa, Y. Fujimoto, H. Ishii, I. Ono, T. Onoda, and Y. Hayashi, "Detection of cyber attacks against voltage control in distribution power grids with PVs," IEEE Transactions on Smart Grid, vol. 7, pp. 1824-1835, July 2016.

[15] L. Gan, N. Li, U. Topcu, and S. H. Low, "Exact convex relaxation of optimal power flow in radial networks," IEEE Transactions on Automatic Control, vol. 60, no. 1, pp. 72-87, 2015.

[16] "IEEE standard for interconnection and interoperability of distributed energy resources with associated electric power systems interfaces," IEEE Std 1547-2018.

[17] "Proofs to theorems and corollaries." https://tinyurl.com/y55gyxph.

[18] R. D. Zimmerman, C. E. Murillo-Sanchez, and R. J. Thomas, "MATPOWER: Steady-state operations, planning, and analysis tools for power systems research and education," IEEE Transactions on Power Systems, vol. 26, pp. 12-19, Feb. 2011.

[19] "Pecan street dataport." https://dataport.cloud. 